\title{
Oral cancer screening: serum Raman spectroscopic approach
}

Aditi K. Sahu Suyash Dhoot

Amandeep Singh

Sharada S. Sawant

Nikhila Nandakumar

Sneha Talathi-Desai

Mandavi Garud

Sandeep Pagare

Sanjeeva Srivastava

Sudhir Nair

Pankaj Chaturvedi

C. Murali Krishna 


\title{
Oral cancer screening: serum Raman spectroscopic approach
}

\author{
Aditi K. Sahu, ${ }^{a}$ Suyash Dhoot, ${ }^{\mathrm{b}}$ Amandeep Singh, ${ }^{\mathrm{c}}$ Sharada S. Sawant, ${ }^{\mathrm{d}}$ Nikhila Nandakumar, ${ }^{\mathrm{a}}$ \\ Sneha Talathi-Desai, ${ }^{a}$ Mandavi Garud, ${ }^{c}$ Sandeep Pagare, ${ }^{c}$ Sanjeeva Srivastava, ${ }^{b}$ Sudhir Nair, ${ }^{e}$ \\ Pankaj Chaturvedi, ${ }^{e}$ and C. Murali Krishna ${ }^{a, *}$ \\ aACTREC-TMC, Chilakapati Lab, Kharghar, Navi Mumbai 410210, India \\ Indian Institute of Technology Bombay, Department of Biosciences and Bioengineering, Powai, Mumbai 400076, India \\ ${ }^{C} D$. Y. Patil Dental College, Nerul, Navi Mumbai 400706, India \\ dACTREC-TMC, Vaidya Laboratory, Kharghar, Navi Mumbai 410210, India \\ 'Tata Memorial Center, Head and Neck Surgical Oncology, Dr. E Borges Road, Parel, Mumbai 400012, India
}

\begin{abstract}
Serum Raman spectroscopy (RS) has previously shown potential in oral cancer diagnosis and recurrence prediction. To evaluate the potential of serum RS in oral cancer screening, premalignant and cancer-specific detection was explored in the present study using 328 subjects belonging to healthy controls, premalignant, disease controls, and oral cancer groups. Spectra were acquired using a Raman microprobe. Spectral findings suggest changes in amino acids, lipids, protein, DNA, and $\beta$-carotene across the groups. A patient-wise approach was employed for data analysis using principal component linear discriminant analysis. In the first step, the classification among premalignant, disease control (nonoral cancer), oral cancer, and normal samples was evaluated in binary classification models. Thereafter, two screening-friendly classification approaches were explored to further evaluate the clinical utility of serum RS: a single four-group model and normal versus abnormal followed by determining the type of abnormality model. Results demonstrate the feasibility of premalignant and specific cancer detection. The normal versus abnormal model yields better sensitivity and specificity rates of 64 and $80 \%$; these rates are comparable to standard screening approaches. Prospectively, as the current screening procedure of visual inspection is useful mainly for high-risk populations, serum RS may serve as a useful adjunct for early and specific detection of oral precancers and cancer. $\odot 2015$ Society of Photo-Optical Instrumentation Engineers (SPIE) [DOI: 10.1117/1.JBO.20.11.115006]
\end{abstract}

Keywords: oral cancer; screening; serum Raman spectroscopy; principal component linear discriminant analysis; serum; blood. Paper 150528R received Aug. 7, 2015; accepted for publication Oct. 9, 2015; published online Nov. 17, 2015.

\section{Introduction}

Screening is an important tool in the overall management of cancers and has led to early detection and decreased mortality rates for several cancers. Screening tests like the Pap test, fecal-occultblood test (FOBT), and mammography have reduced the morbidity and mortality associated with cervix, colorectal, and breast cancers. ${ }^{1-3}$ Oral cancers are the 16 th most common cancers in the world, with lower disease-free survival rates than occult cancers like breast and cervix. It is also the most commonly occurring cancer in Indian males and accounts for $>30 \%$ of all cancers in India. ${ }^{4}$ In spite of the amenability and accessibility of the oral cavity, oral cancers are frequently diagnosed only in advanced stages. ${ }^{5}$ Early detection of oral cancer thus remains the best way to ensure patient survival and improved quality of life. ${ }^{6,7}$ However, no definite screening test is available for oral cancer.

Visual inspection followed by biopsy and histopathology is the gold standard for screening and diagnosis of oral cancers. A study has demonstrated the positive effect of visual screening on oral cancer mortality in a cluster-randomized controlled trial in India in high-risk populations. ${ }^{8}$ Adjunct techniques, like toluidine blue staining, oral cytology, tissue fluorescence (VelScope), and chemiluminescence (Vizilite)-based methods,

*Address for correspondence to: C. Murali Krishna, E-mail: mchilakapati@ actrec.gov.in are being explored as complementary techniques to visual inspection. ${ }^{9}$ Other optical spectroscopic techniques, like infrared (IR), fluorescence, and Raman, have also been explored for oral cancer diagnosis. Raman spectroscopy (RS), a vibrational spectroscopic method based on inelastic scattering of light, ${ }^{10}$ yields a global profile of biochemical changes in the sample. In vivo RS has shown potential in detecting premalignant conditions and cancer field effects, ${ }^{11,12}$ but their screening applications are limited by the need of involved instrumentation and strict experimental conditions.

Blood test-based screening is more practical and desirable, particularly for mass screening of populous nations like India and other developing countries where oral cancer is endemic. Blood-based screening tests, like cancer antigen-125, alpha feto-protein, and prostate specific antigen, have already been employed for early detection of ovarian, liver, and prostate cancer. ${ }^{13-15}$ However, a biomarker exclusive to presence of oral cancer still eludes the scientific and medical community. As an alternative, measurement of global changes in the metabolic profile of a sample may also aid in cancer diagnosis. A recent study that investigates the entire metabolome of blood samples has shown potential in prediction of breast cancer with $80 \%$ efficiency. ${ }^{16}$ Serum RS has previously shown

$1083-3668 / 2015 / \$ 25.00$ @ 2015 SPIE 
potential in detecting cancers like breast, colorectal, ovarian, pancreatic, and cervical cancers. ${ }^{17-22}$ Our previous studies have shown the potential of serum RS in the management of oral cancers. Diagnosis of oral cancers using both resonance and conventional Raman spectroscopy was achieved with a classification efficiency of $\sim 78 \%$ between normal and oral cancer groups. ${ }^{23,24}$ Recurrence in oral cancers was identified by serum RS of postsurgery samples with $\sim 80 \%$ efficiency. ${ }^{25}$ Before envisaging clinical or screening-related applications, the efficacy of serum RS in early precancer (premalignant), specific cancer detection, and evaluation of results on a large sample size is mandatory. Serum RS-based diagnosis of premalignant conditions for oral cancer, is hitherto unreported. Although serum RS has been shown to detect several cancers, the specific detection of any cancer has also not been demonstrated. In the present study, the detection of oral premalignant conditions (leukoplakia, oral submucous fibrosis, and tobacco pouch keratosis) and specific detection of oral cancer using a disease control group (nonoral cancer, i.e., glioma) was first explored. The classification between normal and oral cancer groups was then evaluated on a large cohort. Finally, the overall clinical utility of serum RS was investigated using classification models for screening. A patient-wise approach (where the average spectrum computed from all spectra of a sample/case is employed for data analysis) was employed for analysis. The findings are presented in this paper.

\section{Materials and Methods}

\subsection{Sample Collection Centers}

Subject categories of normal, disease control, oral premalignant, and oral cancer were included in the present study. The subjects for these categories were screened and selected from outpatient department services of Tata Memorial Center, Mumbai, and D. Y. Patil Dental College and Hospital, Navi Mumbai. The normal (healthy or control) subjects were selected from the patients' relatives, friends, and staff members of these institutes. Required ethical clearance was obtained from the respective participating institutions before the start of the study.

\subsection{Sample Accrual}

Subjects who were clinically normal (without any apparent current disease), with or without tobacco/alcohol habits were categorized under the Normal group. The disease control group was included as a nonoral cancer malignancy control. Glioma patients belonging to grades II to IV were included in this group. The oral premalignant category comprised subjects with clinically evident mucosal changes, either homogenous or heterogeneous leukoplakia patch, tobacco pouch keratotic lesion or palpable oral submucous fibrosis (OSMF). The oral cancer category consisted of subjects with primary histopathologically confirmed oral cancer at subsites buccal mucosa or

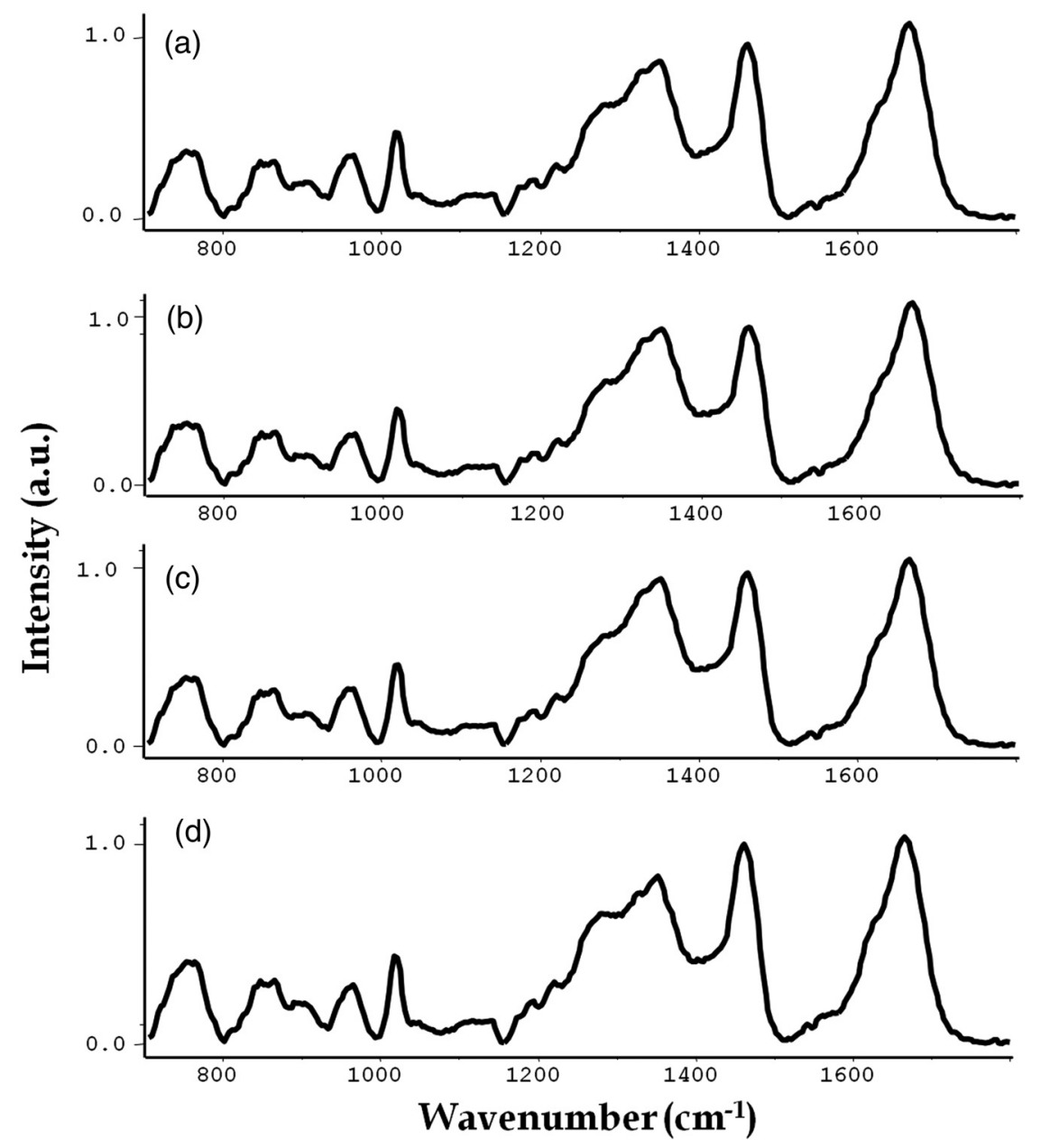

Fig. 1 Average spectra for spectral comparisons: (a) normal, (b) disease control, (c) premalignant, and (d) oral cancer. 
tongue. Patients with a clinical history of anticancer treatment, chemo or radiotherapy, second malignancy, and recurrence, were excluded from the present study. Both oral cancers and oral premalignant subjects were found to have long-term tobacco abuse history. After preliminary screening, serum samples were collected from 328 subjects during 2010 to 2014. Some 126 subjects satisfied the criterion for normal, 120 for oral cancer, 47 for oral premalignant, and 35 for disease control. Written informed consent and demographic information was collected from each subject before sample collection.

\subsection{Sample Collection}

After overnight fasting, $5 \mathrm{ml}$ blood samples were collected by venipuncture in vacuette tubes (Improve vacutainer, Guangzhou Improve Medical Instruments Co. Ltd., China) with clot activator to obtain serum. The tubes were kept standing for $2 \mathrm{~h}$ to allow clot formation, and centrifuged at $3000 \mathrm{rpm}$ for $10 \mathrm{~min}$. The supernatant was separated, aliquoted, snap frozen in liquid nitrogen, and finally stored at $-80^{\circ} \mathrm{C}$ until Raman analysis.

\subsection{Raman Spectroscopy}

After passive thawing on ice, a $30 \mu 1$ volume of sample was placed on calcium fluoride $\left(\mathrm{CaF}_{2}\right)$ window and spectra were acquired using a fiber-optic Raman microprobe. Details of the instrument have been described previously. ${ }^{26}$ Briefly, the system consists of a laser (785 nm, Process Instruments) as an excitation source and HE 785 spectrograph (HoribaJobin-Yvon, France) coupled with a charge coupled device (CCD) (Synapse, Horiba-Jobin-Yvon) as dispersion and detection elements, respectively. Optical filtering of unwanted noise, including Rayleigh signals, is accomplished through the superhead. The Raman microprobe was assembled by coupling a $40 \times$ microscopic objective (Nikon, Japan) to the superhead. Spectral acquisition details were as follows: $\lambda_{\text {ex }}: 785 \mathrm{~nm}$; laser power: $30 \mathrm{~mW}$; integration time: $15 \mathrm{~s}$; and average: 4 . On an average, seven spectra were recorded from each sample. Mean spectra were calculated by averaging all spectra of a sample: a patient-wise approach was employed for data analysis.

Table 1 Principal component linear discriminant analysis (PC-LDA) for classification between normal and premalignant serum samples [leave-one-out cross-validation (LOOCV) confusion matrix results for the analysis].

\begin{tabular}{lccc} 
& Normal & Premalignant & Total \\
\hline Normal & 95 & 31 & 126 \\
Premalignant & 10 & 37 & 47 \\
\hline
\end{tabular}

(a)
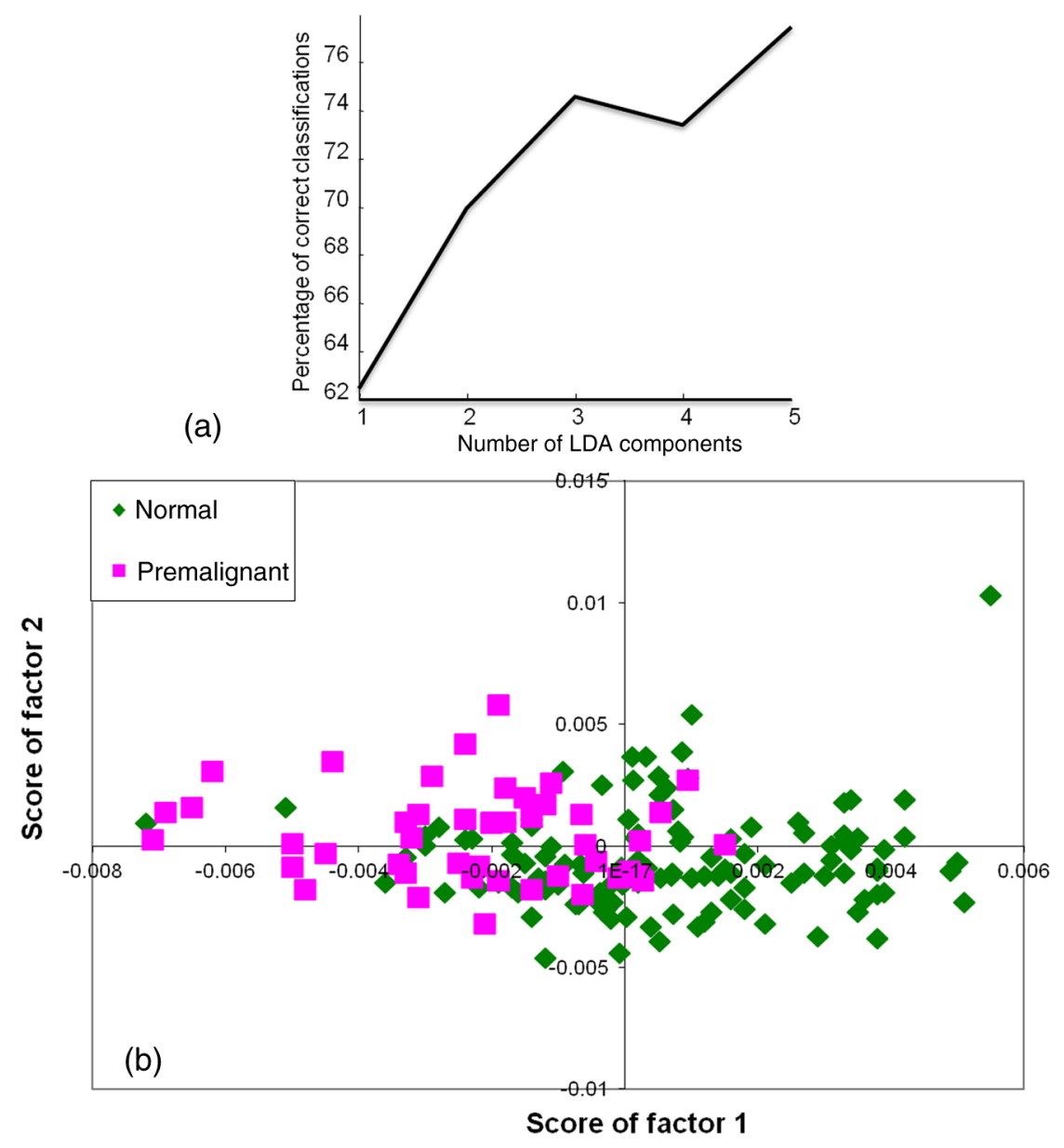

Fig. 2 Principal component linear discriminant analysis (PC-LDA) for normal and premalignant subjects: (a) scree plot and (b) scatter plot. 


\subsection{Spectral Preprocessing and Data Analysis}

Spectra were corrected for CCD response with a National Institute of Science and Technology certified Standard Reference Material 2241 followed by the subtraction of background signals from optical elements and substrate. To remove interference of the slow moving background, first derivatives of spectra (Savitzky-Golay method and window size 3) were computed. $^{27,28}$ Spectra were interpolated in the 700 to $1800 \mathrm{~cm}^{-1}$ region, vector-normalized, and used as input for multivariate analysis. Multivariate tool principal component linear discriminant analysis (PC-LDA) followed by leave-one-out cross-validation (LOOCV) was used for data analysis.

Average spectra were also computed for spectral comparisons across the groups using the background-corrected spectra prior to derivatization. These spectra were baseline corrected by fitting a fifth-order polynomial function, smoothed (SavitzkyGolay, 3), vector-normalized, and used for spectral comparisons.

\section{Results and Discussion}

Currently practiced modalities for screening of oral cancers include visual inspection, followed by biopsy and histopathology of suspicious lesions. Serum Raman spectroscopy-based screening may serve as a useful adjunct to existing approaches. In the present study, first the feasibility to identify premalignant conditions and specific cancer detection was investigated and differences between oral cancer and normal conditions were evaluated using a large sample size. Finally, screening-related applications of serum RS were verified using two specialized classification systems. Based on previous studies, PC-LDA using the patient-wise approach in the fingerprint region was employed for data analysis.

\subsection{Spectral Analysis}

Mean spectra were computed by averaging background-subtracted spectra prior to derivatization for each class, by averaging $Y$-axis variations keeping the $X$ axis constant for each class, and baseline corrected by fitting a fifth-order polynomial function. The smoothed (Savitzky-Golay method, 3), normalized average spectra for all groups are shown in Figs. 1(a)-1(d). Major spectral features were observed at 830 and 850 (Tyr doublet), 1007 (Phe), 1085 to 1100 (DNA backbone), 1162 and 1530 ( $\beta$-carotene), 1267 (amide III), 1318 and 1342 (DNA bases), 1451 ( $\mathrm{CH}_{2}$ bending), 1556 and 1622 (Trp), and $1656 \mathrm{~cm}^{-1}$ (amide I). Tentative assignments have been made as per the available literature. ${ }^{29,30}$ Thus, spectra are characterized by the presence of contributions mainly from amino acids, beta carotene, DNA, and proteins. Intensity-related variations in

Table 2 PC-LDA for classification between normal and disease control serum samples (LOOCV confusion matrix results for the analysis).

\begin{tabular}{lccc} 
& Normal & Disease & Total \\
\hline Normal & 116 & 10 & 126 \\
Disease & 5 & 30 & 35 \\
\hline
\end{tabular}

(a)
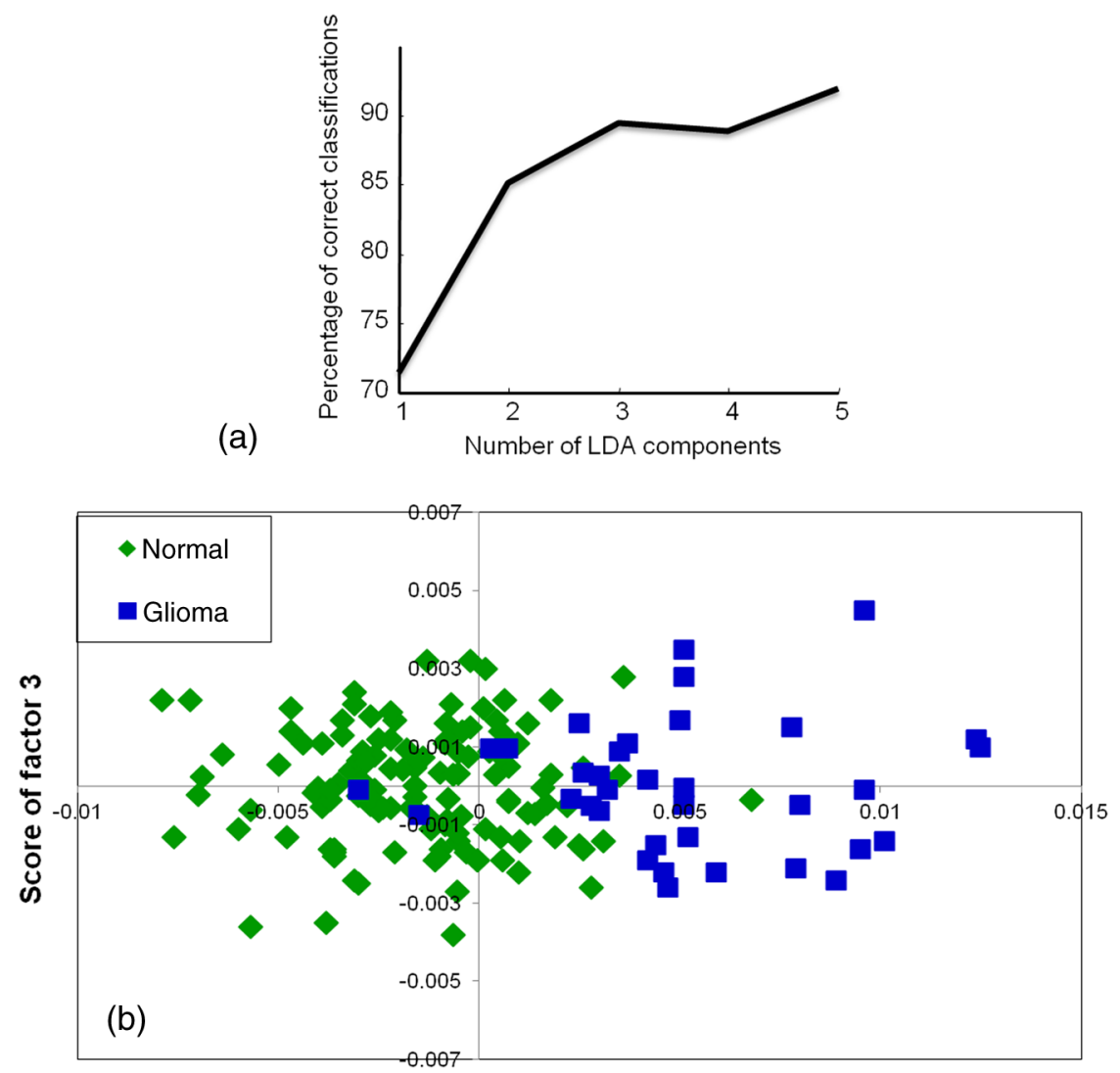

Score of factor 1

Fig. 3 PC-LDA for normal and disease control subjects: (a) scree plot and (b) scatter plot. 
amino acids, amide III, and DNA peaks were observed across the different groups.

Oral cancer spectra showed higher Tyr, Trp (doublet at 830 and 850, 1552), amide III (1270), and $\mathrm{CH}_{2}$ deformation (1450) but slightly lower amide I (1660) and sharper DNA bases (1342). Most of these spectral variations corroborate our previous findings. ${ }^{23,24}$ With respect to the normal group, premalignant spectra showed higher Phe (1008), lower amide III (1270), higher DNA bases (1320, 1342), higher $\mathrm{CH}_{2}$ deformation (1450), and slightly lower amide I (1660). The disease control spectrum showed higher Phe (1008), amide III (1270), DNA bases features $(1320,1342)$, lower $\mathrm{CH}_{2}$ deformation (1450), and higher amide $\mathrm{I}\left(1660 \mathrm{~cm}^{-1}\right)$. It has been reported that the appearance of premalignant changes in the oral cavity is associated with an increase in serum concentrations of enzyme lactate dehydrogenase, $\beta_{2}$-microglobulin, changes in lipid profile; serum glyco-conjugates, like sialic acid; lipid-bound sialic acid; serum antioxidants, like vitamin $\mathrm{A}, \mathrm{E}, \beta$-carotene; and trace elements, like iron, zinc, copper. ${ }^{31-33}$ In the case of gliomas, changes in proteomic profiles; serum gangliosides; trace elements, like copper, zinc, lead, manganese, cobalt; and increase in YKL-40 levels have been linked with its development. ${ }^{34-37}$

\subsection{Multivariate Analysis}

The feasibility of classifying normal, premalignant, oral cancer, and disease control (nonoral cancer) groups using binary classification systems was first explored. PC-LDA was employed to build the standard models followed by cross-validation using LOOCV. In the subsequent step, the potential of serum RS in screening-related applications was investigated using two models: a single all-encompassing four-model and a normal versus abnormal coupled with type of abnormality deduction model.

Table 3 PC-LDA for classification between normal and oral cancer serum samples.

(a) LOOCV confusion matrix normal versus oral cancer

\begin{tabular}{lccc}
\hline & Normal & Cancer & Total \\
\hline Normal & 113 & 13 & 126 \\
Cancer & 19 & 101 & 120 \\
\hline
\end{tabular}

(b) LOOCV of standard model for normal versus oral cancer

\begin{tabular}{lccc}
\hline & Model N & Model C & Total \\
\hline Model N & 53 & 7 & 60 \\
Model C & 10 & 50 & 60 \\
\hline
\end{tabular}

(c) Test prediction using independent test data

\begin{tabular}{lccc} 
& Model N & Model C & Total \\
\hline Test N & 57 & 9 & 66 \\
Test C & 8 & 52 & 60 \\
\hline
\end{tabular}

(a)
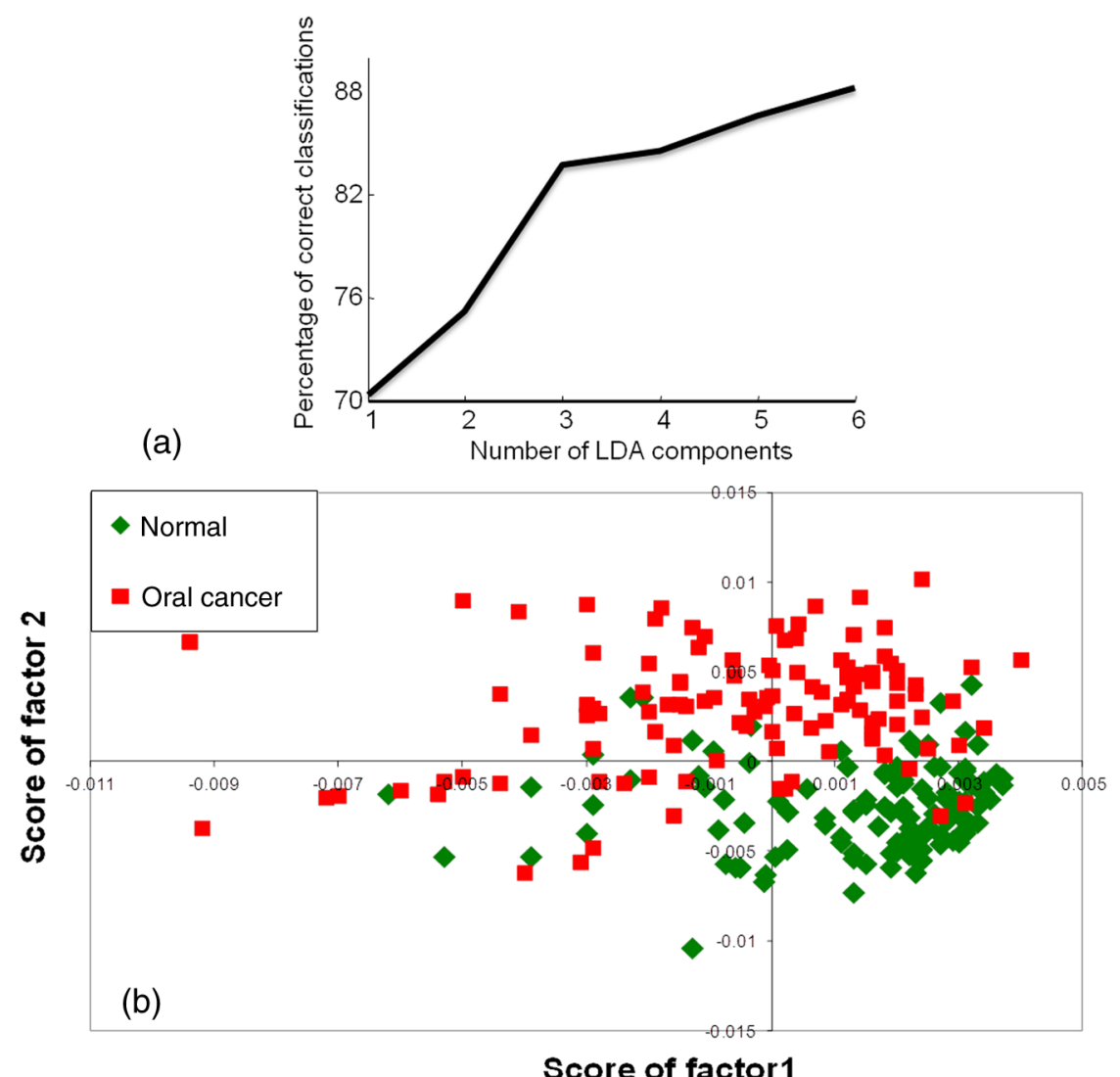

Fig. 4 PC-LDA for normal and oral cancer subjects: (a) scree plot and (b) scatter plot. 


\subsubsection{Exploring classification between normal and oral premalignant groups}

PC-LDA for normal versus premalignant subjects was carried out using five factors (77\% classification efficiency) as shown in Fig. 2(a). The scatter plot indicates slightly overlapping clusters for normal and premalignant groups [Fig. 2(b)]. A lower efficiency for identifying normal $(\sim 75 \%)$ and premalignant conditions $(\sim 79 \%)$ was observed (Table 1$)$. This indicates a similarity in the normal and premalignant serum samples, in light of the lack of appreciable cancer-related changes.

\subsubsection{Exploring classification between normal and disease control groups}

PC-LDA for normal versus disease control was carried out using five factors [Fig. 3(a)]. The PC-LDA scatter plot shown in Fig. 3(b) indicates two almost distinct clusters for normal and glioma. The LOOCV confusion matrix in Table 2 indicates $\sim 89 \%$ overall classification efficiency for identifying both disease and normal samples. Thus, healthy and disease samples can be identified with high sensitivity and specificity.

\subsubsection{Exploring classification between normal and oral cancer groups}

PC-LDA of normal versus oral cancer was carried out using six factors [Fig. 4(a)]. The scatter plot shown in Fig. 4(b) demonstrates two distinct clusters each for normal and oral cancer. Table 3(a) indicates an LOOCV efficiency of $90 \%$ to identify normal and $84 \%$ for detection of cancer samples. To substantiate these findings, a standard model of normal versus oral cancer was built using $n=60$ samples. The remaining 126 samples of normal and oral cancer were used as the independent

Table 4 PC-LDA for exploring cancer-specific detection for oral cancers (LOOCV confusion matrix results for the analysis).

(a) Oral cancer versus glioma

\begin{tabular}{lccc}
\hline & Disease & Oral cancer & Total \\
\hline Disease & 31 & 4 & 35 \\
Oral cancer & 13 & 107 & 120 \\
\hline
\end{tabular}

(b) Normal versus glioma versus oral cancer

\begin{tabular}{lcccc}
\hline & Normal & Disease & Oral cancer & Total \\
\hline Normal & 100 & 15 & 11 & 126 \\
Disease & 8 & 27 & 0 & 35 \\
Oral cancer & 17 & 13 & 90 & 120 \\
\hline
\end{tabular}

(a)
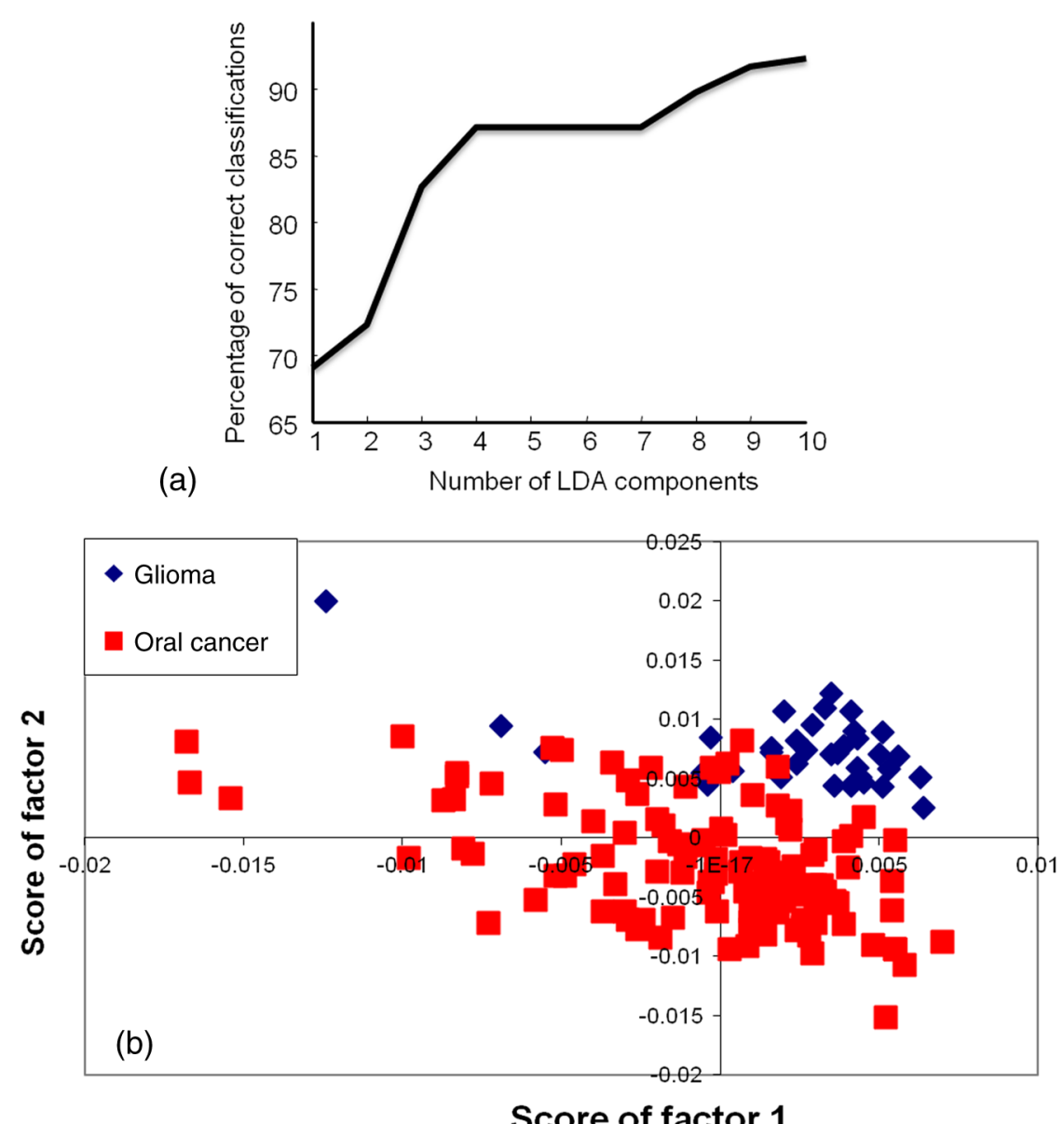

Fig. 5 PC-LDA for disease control and oral cancer subjects: (a) scree plot and (b) scatter plot. 
test dataset on this model. The LOOCV confusion matrix for the standard model and the test predictions are shown in Tables 3(b) and 3(c), respectively. In the standard model, normal and cancer could be identified with 88 and $83 \%$ efficiency, respectively. As shown in Table 3(c), 57/66 normal samples (86\%) and 52/60 cancer samples $(87 \%)$ were predicted correctly. Thus, test predictions indicate that normal and oral cancer samples can be identified with high sensitivity and specificity.

\subsubsection{Exploring cancer-specific diagnosis for oral cancers}

Our previous studies have shown successful classification between normal and oral cancer samples. To determine if RS can differentiate between two different types of cancers, a nonoral cancer group, i.e., glioma, was incorporated in this study. Additionally, specific diagnosis of cancers is important during cancer screening. Thus, the feasibility of differentiating these two different types of cancers was also explored in this study. Oral cancers included in this study are oral squamous cell carcinomas, while gliomas are brain cancers that originate in the glial cells. Different serum biochemistry changes are reported in these cancers, as previously mentioned in the spectral analysis sections. The PC-LDA of oral cancer versus disease control (glioma) samples was first carried out using 10 factors to explore cancer-specific differences in serum [Fig. 5(a)]. The scatter plot shown in Fig. 5(b) shows two distinct clusters for oral cancer and glioma. The LOOCV table [Table 4(a)] demonstrates correct predictions of $31 / 35$ glioma samples and $107 / 120$ oral cancer samples. Thus, these cancers were discriminated with $\sim 89 \%$ efficiency. The distinction between normal and cancer, and simultaneous detection of type of cancer (oral cancer or glioma), was also evaluated using a threemodel system. PC-LDA was carried out using 10 factors accounting for $\sim 79 \%$ efficiency [Fig. 6(a)]. The PC-LDA scatter plot shows three almost distinct clusters corresponding to normal, oral cancer, and disease control [Fig. 6(b)]. The LOOCV confusion matrix shown in Table 4(b) demonstrates the feasibility of identifying normal samples with $\sim 80 \%$ efficiency. Glioma and oral cancer could be identified with 77 and $75 \%$ efficiency, respectively. These results indicate that different cancers have different Raman spectroscopic signatures and may be identified distinctly based on serum spectral characteristics.

\subsubsection{Exploring serum Raman spectroscopy in screening applications}

Two classification models were employed to evaluate the potential of serum RS in oral cancer screening: first, a four-group model, and second, a normal versus abnormal model followed by determining the type of abnormality.

(a)
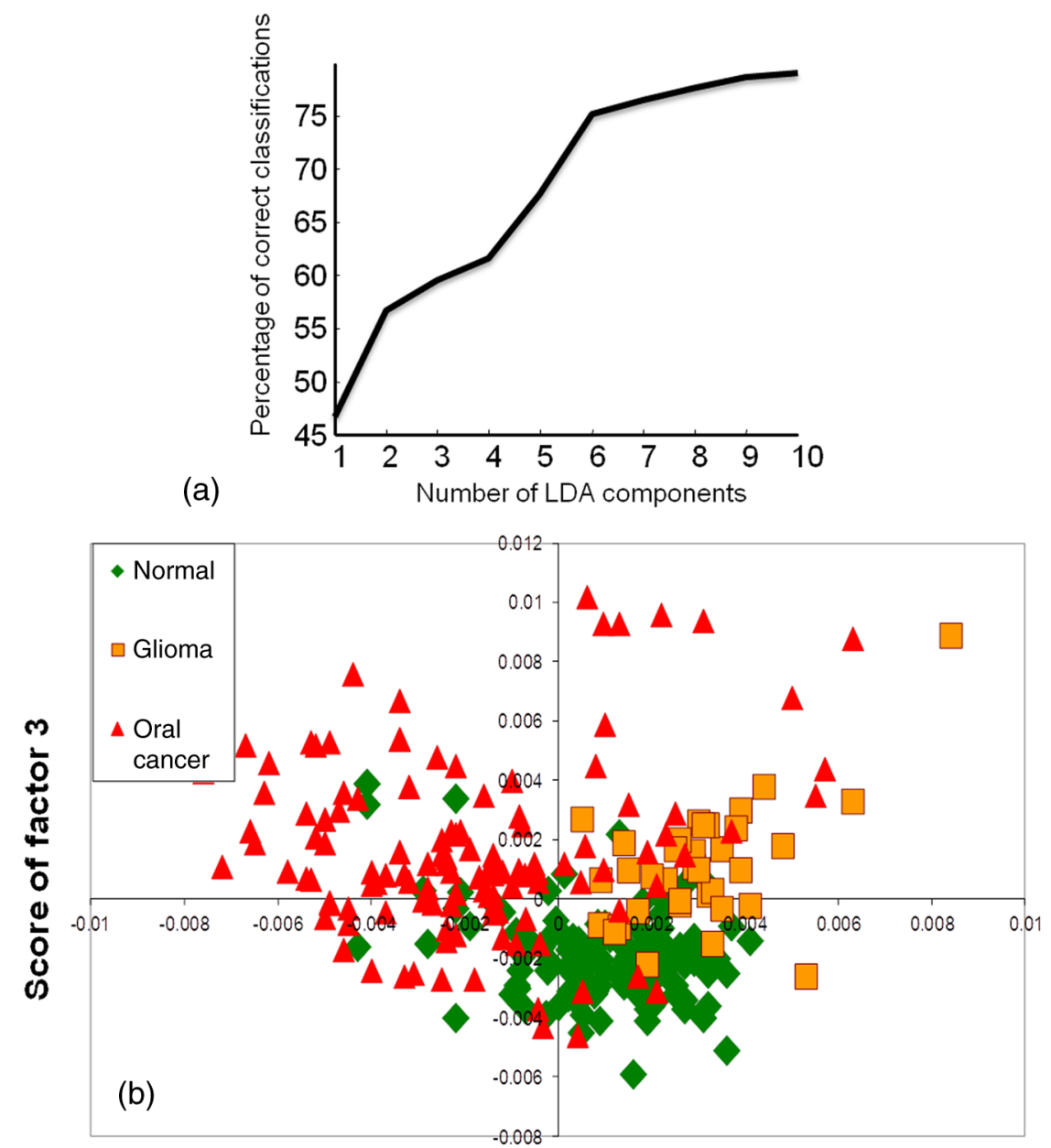

Score of factor 2

Fig. 6 PC-LDA for normal, disease control, and oral cancer subjects: (a) scree plot and (b) scatter plot. 
Exploring classification among normal, disease control, oral premalignant, and cancer sera using a four-model system. In the first screening model, a four-group model approach was used to explore classification among normal, disease control, premalignant, and oral cancer. This is the ideal model for screening applications, as all possible groups constitute this model. Thus, a single-step analysis will be required to ascertain the identity of the sample during screening. PC-LDA was carried out using seven factors, which accounted for $~ 64 \%$ correct classifications. The scree plot and PC-LDA scatter plot are shown in Fig. 7. As shown in Table 5, several misclassifications among normal, disease, and premalignant groups were observed. About 15 and 25\% normal misclassified with disease and premalignant, respectively, while only $10 \%$ misclassified with the cancer group. More number of normal misclassifications observed with premalignant group could be due to less severe biochemical changes as compared to cancer groups. In case of cancer, $15 \%$ were misclassified with normal, $10 \%$ with disease, and $8 \%$ with premalignant groups. Thus, overall, a specificity of $\sim 90 \%$ and sensitivity of $\sim 85 \%$ was observed for normal versus oral cancer classification. Premalignant and disease controls could also be identified with 71 and $66 \%$ efficiency.

Exploring classification between normal and abnormal. In the second classification model, the feasibility to identify a healthy/normal serum sample against all other pathological/abnormal conditions was explored. Thus, in this first
Table 5 Standard model for screening applications I-single fourgroup model for classification among normal, disease control, premalignant, and oral cancer (LOOCV confusion matrix results for the analysis).

Normal Disease Premalignant Oral cancer Total

\begin{tabular}{lccccc}
\hline Normal & 63 & 17 & 33 & 13 & 126 \\
Disease & 7 & 25 & 3 & 0 & 35 \\
Premalignant & 11 & 5 & 31 & 0 & 47 \\
Oral cancer & 17 & 11 & 9 & 83 & 120 \\
\hline
\end{tabular}

step, classification between the normal group and a combined abnormal group (disease + premalignant + oral cancer) was explored. In this classification system, if a sample would be classified as abnormal, the second step would be carried out to identify the type of abnormality: disease, premalignant, or malignant. This could be an ideal approach for screening-related applications where healthy samples are excluded and abnormal samples are subjected to further round of confirmatory analyses. The first normal versus abnormal PC-LDA classification was carried out using six factors accounting for $71 \%$ classification efficiency [Fig. 8(a)]. The scatter plot shown in Fig. 8(b) indicates two almost distinct clusters corresponding to normal and abnormal. As shown in Table 6(a), normal could be identified

(a)
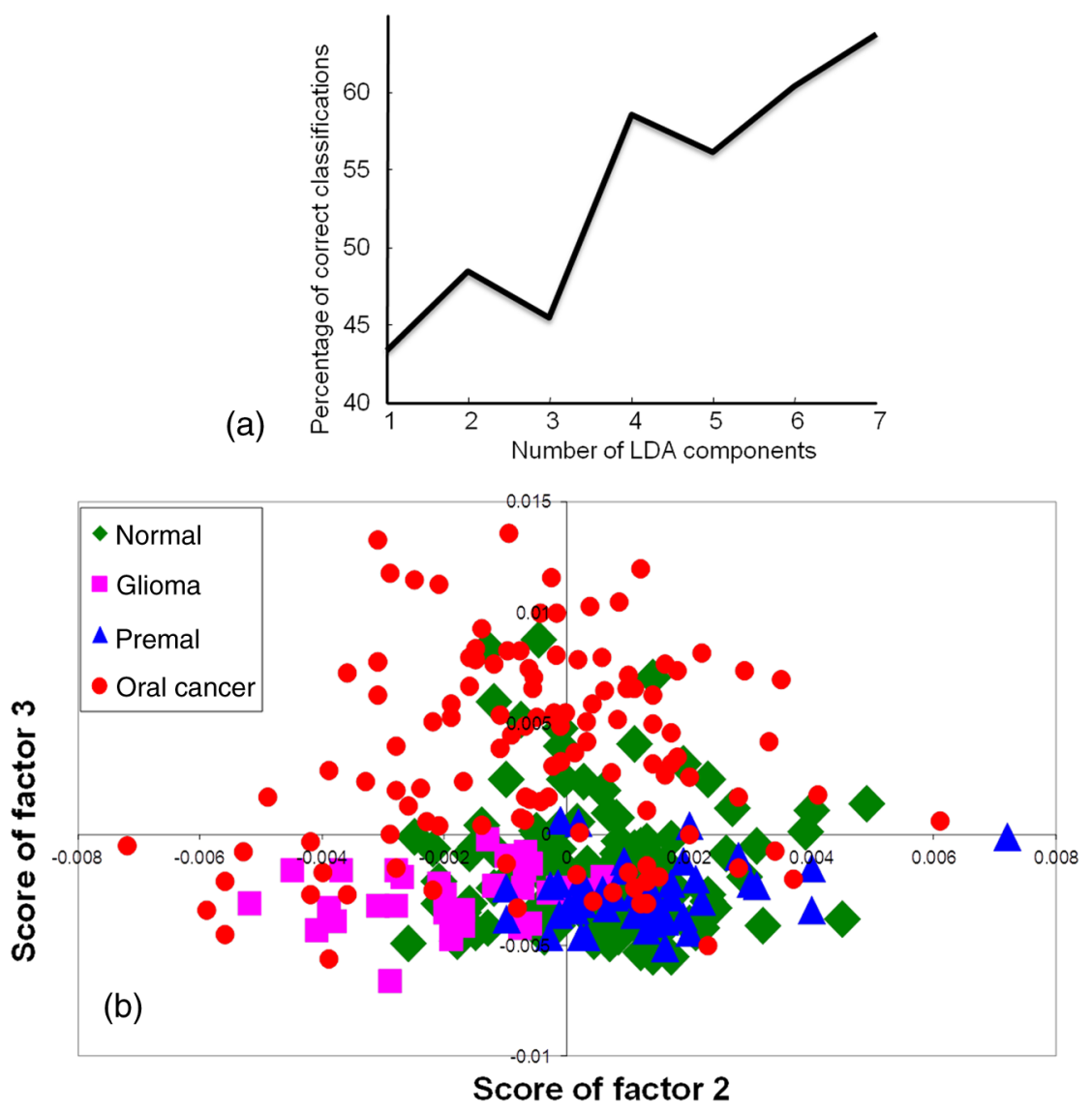

Fig. 7 PC-LDA for normal, premalignant, disease control, and oral cancer: (a) scree plot and (b) scatter plot. 
(a)
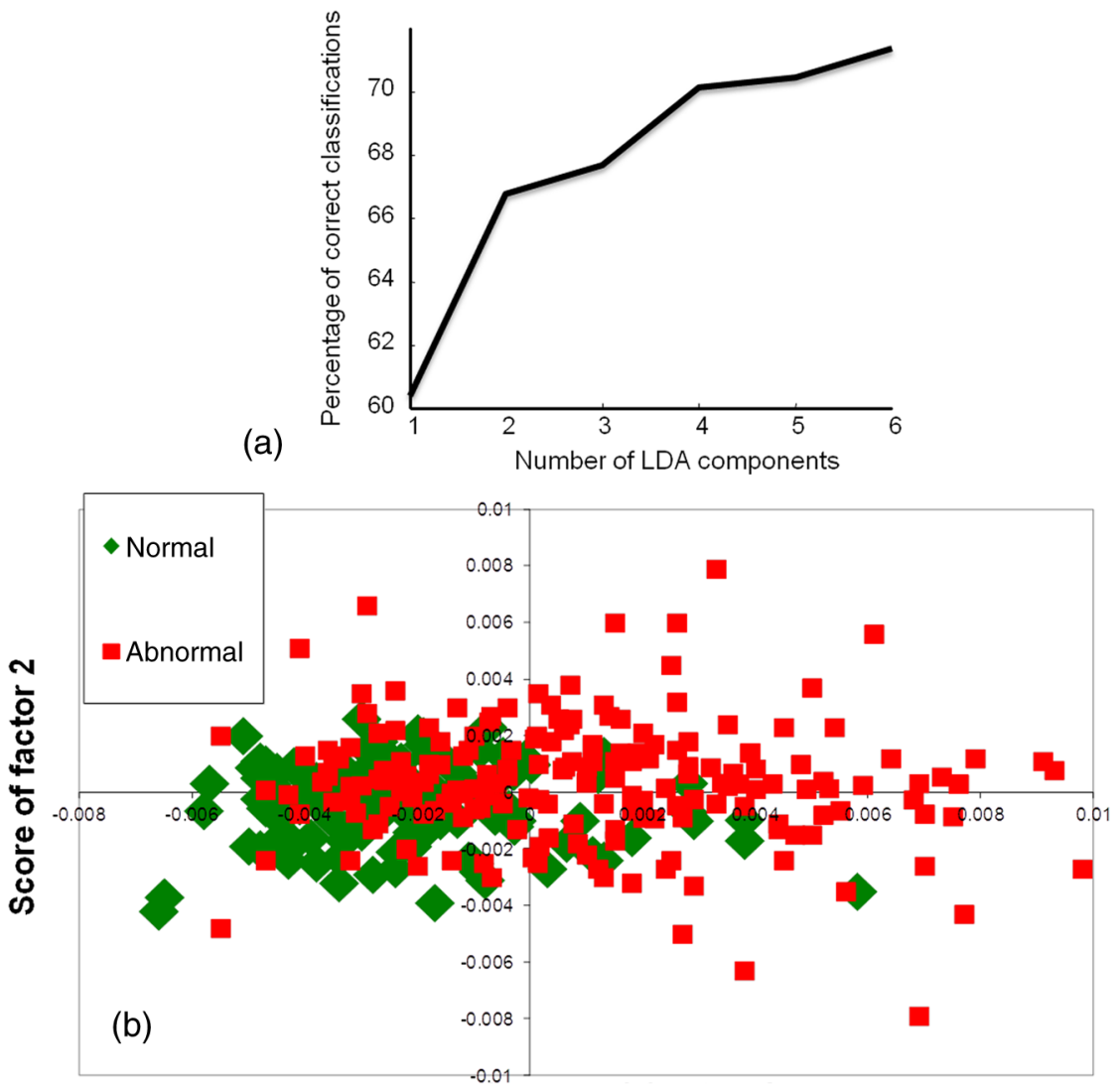

Score of factor 1

Fig. 8 PC-LDA for normal and all abnormal subjects: (a) scree plot and (b) scatter plot.

with $\sim 80 \%$ efficiency, while abnormal or pathological could be identified with $64 \%$ efficiency. Thus, the sensitivity and specificity for this approach was found to be $\sim 64$ and $\sim 80 \%$, respectively. The feasibility to differentiate the different abnormal conditions was explored in the next step. PC-LDA was carried out using seven factors accounting for $\sim 78 \%$ efficiency [Fig. 9(a)]. The scatter plot shown in Fig. 9(b) indicates two

Table 6 Standard model for screening applications II-normal versus abnormal followed by determination of type of abnormality (LOOCV confusion matrix results for the analysis).

(a) Normal versus all abnormal

\begin{tabular}{lccc}
\hline & Normal & Abnormal & Total \\
\hline Normal & 101 & 25 & 126 \\
Abnormal & 73 & 129 & 202 \\
\hline
\end{tabular}

(b) Glioma versus premalignant versus oral cancer

\begin{tabular}{lcccc}
\hline & Glioma & Premalignant & Oral cancer & Total \\
\hline Glioma & 26 & 8 & 1 & 35 \\
Premalignant & 9 & 36 & 2 & 47 \\
Oral cancer & 14 & 15 & 91 & 120 \\
\hline
\end{tabular}

overlapping clusters for disease control and premalignant while a slightly distinct cluster for oral cancer. In total, 26/35 disease subjects could be correctly classified, while $36 / 47$ premalignant and 91/120 cancers were correctly classified [Table 6(b)]. The sensitivity to detect each abnormal condition was 74,79 , and $75 \%$ for disease, premalignant, and cancer, respectively. Most disease misclassifications were observed with premalignant samples and vice versa. Few premalignant (2/47) and malignant (15/120) misclassifications were observed between these two classes. The two premalignant subjects classified as malignant may be indicative of imminent malignant transformation, though no follow-up of these subjects was conducted in the present study. The lower rates of identifying premalignant as cancer could be attributed to the fact that only $4 \%$ leukoplakia and 5 to $10 \%$ OSMF transform into malignancy. ${ }^{6,38}$ During screening applications, such samples can nevertheless be termed as suspicious and followed up for malignancy after primary screening. Overall, in this analysis, disease could be identified with $74 \%$, premalignant with $77 \%$, and oral cancer with $\sim 76 \%$ efficiency. But the identification of abnormal condition against normal in the first step had a lower sensitivity rate of $\sim 64 \%$ and higher specificity rate of $80 \%$. For an ideal cancer screening test, higher sensitivity is desirable to eliminate the possibility of losing any malignant cases to false negative diagnosis. Although the lower specificity rates may also be a concern, the samples falsely classified as positives will only be treated as suspicious, and all these suspiciously abnormal samples will be deemed as cancer only after several rounds of confirmatory tests. 
(a)
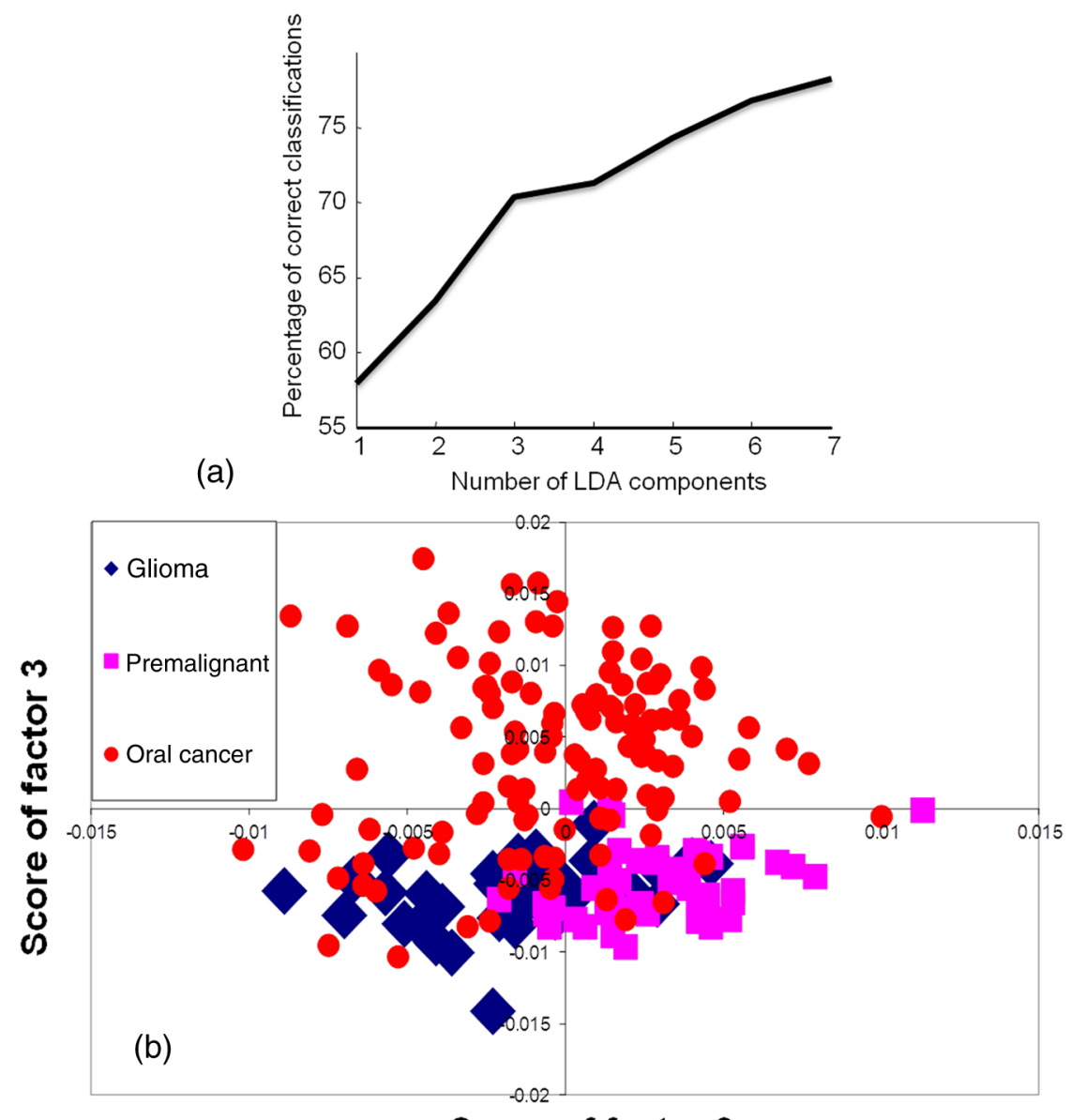

Score of factor 2

Fig. 9 PC-LDA for abnormal conditions premalignant, disease control, and oral cancer: (a) scree plot and (b) scatter plot.

\section{Conclusions}

Screening of cancers using a peripheral blood sample may be a practical and feasible approach. This approach is associated with multiple advantages like accessibility, low invasive procedure, low cost, and multiple sampling. ${ }^{39}$ Moreover, samples can be transported from primary screening centers to a centralized facility for analysis. Recent studies have shown the utility of blood sample-based liquid biopsy in cancer diagnosis and monitoring treatment response by detecting DNA fragments, or by detecting blood telomere length for predicting the onset of cancer. ${ }^{40,41}$ In cancers where definite biomarkers are not available, the global biochemical changes can be identified by spectroscopic techniques like nuclear magnetic resonance (NMR), IR, fluorescence, and RS. A more recent study has shown to predict breast cancer five years before its occurrence with $80 \%$ sensitivity, using metabolic changes in serum by NMR spectroscopy. ${ }^{16}$ Serum RS had previously shown potential in detecting several diseases, cancers, monitoring treatment response, and predicting recurrence. ${ }^{25}$ In the present study, the efficacy of serum RS in detecting premalignant conditions, specific detection of oral cancers, and utility in oral cancer screening were evaluated. While the premalignant samples could be identified with $\sim 75 \%$ efficiency, normal and disease controls could be identified with a higher efficiency of $\sim 89 \%$. The analysis on a large sample size and independent test data evaluation confirms the discrimination between normal and oral cancer groups with an average efficiency of $\sim 86 \%$.

Screening applications were further explored by using two classification models. While the first four-group model yielded reasonable efficiency of classification, the normal versus abnormal model yielded sensitivity of $64 \%$ and specificity of $\sim 80 \%$. Although the sensitivity and specificity rates are not very high, they are quite comparable to established screening techniques like FOBT, Pap smear, and mammography. ${ }^{42-44}$ In spite of medium sensitivity $(67.8 \%)$ and specificity (75\%) and other limitations, mammography remains the most preferred approach for breast cancer screening. Even using Pap smears, where the sensitivity of a single Pap smear is reported to be between 60 and $80 \%$, screening has reduced the incidence and mortality of invasive cervical cancers by $\sim 90 \%{ }^{45}$ As the current screening approaches (visual inspection followed by biopsy and histopathology) are useful mainly for high-risk populations, ${ }^{8}$ oral cancer screening using serum RS may serve as useful adjuncts and help in improving low disease-free survival rates associated with oral cancers. Other approaches like Raman mapping and sample concentration methods like drop coating deposition Raman may improve the overall sensitivity rates of serum RS-based screening. Before envisaging clinical applications, a more rigorous validation using various population cohorts, several disease controls, and more premalignant cases are warranted. 


\section{Acknowledgments}

The Raman spectrometer employed in the study was procured from DBT project BT/PRI11282/MED/32/83/2008, titled "Development of in vivo laser Raman spectroscopy methods for diagnosis of oral precancerous and cancerous conditions," Department of Biotechnology, Government of India. The authors would like to acknowledge technicians and nurses of Tata Memorial Center for help in blood collection.

\section{References}

1. B. Levin et al., "Screening and surveillance for the early detection of colorectal cancer and adenomatous polyps, 2008: a joint guideline from the American Cancer Society, the US Multi-Society Task Force on Colorectal Cancer, and the American College of Radiology," CA Cancer J. Clin. 58(3), 130-160 (2008).

2. D. Saslow et al., "American Cancer Society guidelines for breast screening with MRI as an adjunct to mammography," CA Cancer J. Clin. 57(2), 75-89 (2007).

3. D. Saslow, D. Solomon, and H. Lawson, "Screening guidelines for the prevention and early detection of cervical cancer," CA Cancer J. Clin. 62(3), 147-172 (2012).

4. K. R. Coelho, "Challenges of the oral cancer burden in India," J. Cancer Epidemiol. 2012, 701932 (2012).

5. S. Khandekar, P. Bagdey, and R. Tiwari, "Oral cancer and some epidemiological factors: a hospital based study," Ind. J. Commun. Med. 31(3), 157-159 (2006).

6. S. S. Napier and P. M. Speight, "Natural history of potentially malignant oral lesions and conditions: an overview of the literature," J. Oral Pathol. Med. 37(1), 1-10 (2008).

7. I. van der Waal, "Potentially malignant disorders of the oral and oropharyngeal mucosa; present concepts of management," Oral Oncol. 46(6), 423-425 (2010).

8. R. Sankaranarayanan et al., "Effect of screening on oral cancer mortality in Kerala, India: a cluster-randomised controlled trial," Lancet 365(9475), 1927-1933 (2005).

9. D. V. Messadi, "Diagnostic aids for detection of oral precancerous conditions," Int. J. Oral Sci. 5(2), 59-65 (2013).

10. C. V. Raman and K. S. Krishnan, "A new type of secondary radiation," Nature 121(3048), 501-502 (1928).

11. S. P. Singh et al., "In vivo Raman spectroscopic identification of premalignant lesions in oral buccal mucosa," J. Biomed. Opt. 17(10), 105002 (2012).

12. S. Singh et al., "In vivo Raman spectroscopy of oral buccal mucosa: a study on malignancy associated changes (MAC)/cancer field effects (CFE)," Analyst 138(14), 4175-4182 (2013).

13. U. Menon et al., "Sensitivity and specificity of multimodal and ultrasound screening for ovarian cancer, and stage distribution of detected cancers: results of the prevalence screen of the UK Collaborative Trial of Ovarian Cancer Screening (UKCTOCS)," Lancet Oncol. 10(4), 327-340 (2009).

14. B. Daniele et al., " $\alpha$-fetoprotein and ultrasonography screening for hepatocellular carcinoma," Gastroenterology 127(5), S108-S112 (2004).

15. R. Harris and K. N. Lohr, "Screening for prostate cancer: an update of the evidence for the US Preventive Services Task Force," Ann. Intern. Med. 137(11), 917-929 (2002).

16. R. Bro et al., "Forecasting individual breast cancer risk using plasma metabolomics and biocontours," Metabolomics 11(5), 1-5 (2015).

17. J. Pichardo-Molina et al., "Raman spectroscopy and multivariate analysis of serum samples from breast cancer patients," Lasers Med. Sci. 22(4), 229-236 (2007).

18. D. Lin et al., "Colorectal cancer detection by gold nanoparticle based surface-enhanced Raman spectroscopy of blood serum and statistical analysis," Opt. Express 19(14), 13565-13577 (2011).

19. S. Feng et al., "Nasopharyngeal cancer detection based on blood plasma surface-enhanced Raman spectroscopy and multivariate analysis," Biosensors Bioelectron. 25(11), 2414-2419 (2010).

20. J. L. González-Solís et al., "Cervical cancer detection based on serum sample Raman spectroscopy," Lasers Med. Sci. 29(3), 979-985 (2014).
21. G. L. Owens et al., "Vibrational biospectroscopy coupled with multivariate analysis extracts potentially diagnostic features in blood plasma/serum of ovarian cancer patients," J. Biophotonics 7(3-4), 200-209 (2014).

22. G. Wang et al., "Detection of the potential pancreatic cancer marker MUC4 in serum using surface-enhanced Raman scattering," Anal. Chem. 83(7), 2554-2561 (2011).

23. A. Sahu et al., "Raman spectroscopy of serum: an exploratory study for detection of oral cancers," Analyst 138(14), 4161-4174 (2013).

24. A. Sahu et al., "Raman spectroscopy of serum: a study on oral cancers," Biomed. Spectrosc. Imaging 4(2), 171-187 (2015).

25. A. Sahu et al., "Recurrence prediction in oral cancers: a serum Raman spectroscopy study," Analyst 140(7), 2294-2301 (2015).

26. A. Sahu et al., "Serum based diagnosis of asthma using Raman spectroscopy: an early phase pilot study," PLOS One 8(11), e78921 (2013).

27. S. Koljenović et al., "Discriminating vital tumor from necrotic tissue in human glioblastoma tissue samples by Raman spectroscopy," Lab. Invest. 82(10), 1265-1277 (2002).

28. A. Nijssen et al., "Discriminating basal cell carcinoma from perilesional skin using high wave-number Raman spectroscopy," J. Biomed. Opt. 12(3), 034004 (2007).

29. F. S. Parker, Applications of Infrared, Raman, and Resonance Raman Spectroscopy in Biochemistry, Springer Science \& Business Media, New York (1983).

30. Z. Movasaghi, S. Rehman, and I. U. Rehman, "Raman spectroscopy of biological tissues," Appl. Spectrosc. Rev. 42(5), 493-541 (2007).

31. A. P. Singh et al., "Estimation of serum $\beta 2$-microglobulin in potentially malignant disorders and squamous cell carcinoma of the oral cavity: a clinicopathological study," Dent. Res. J. 11(1), 109 (2014).

32. V. Kamath, K. Satelur, and Y. Komali, "Biochemical markers in oral submucous fibrosis: a review and update," Dent. Res. J. 10(5), 576 (2013).

33. T. Pereira, S. Shetty, and S. Pereira, "Estimation of serum lactate dehydrogenase level in patients with oral premalignant lesions/conditions and oral squamous cell carcinoma: a clinicopathological study," J. Cancer Res. Ther. 11(1), 78 (2015).

34. F. M. Iwamoto et al., "Serum YKL-40 is a marker of prognosis and disease status in high-grade gliomas," Neuro Oncol. 13(11), 1244-1251 (2011).

35. B. Radić, Ž. Vukelić, and S. Kalanj Bognar, "Serum gangliosides in patients with brain tumors," Coll. Antropol. 32(1), 171-175 (2008).

36. M. Arslan et al., "Trace elements, heavy metals and other biochemical parameters in malignant glioma patients," Asian Pac. J. Cancer Prev. 12(2), 447-451 (2011).

37. I. D. Popescu et al., "Potential serum biomarkers for glioblastoma diagnostic assessed by proteomic approaches," Proteome Sci. 12(1), 1-14 (2014).

38. P.-S. Ho et al., "Malignant transformation of oral potentially malignant disorders in males: a retrospective cohort study," BMC Cancer 9(1), 260 (2009).

39. D. M. Good et al., "Body fluid proteomics for biomarker discovery: lessons from the past hold the key to success in the future," J. Proteome Res. 6(12), 4549-4555 (2007).

40. M. Roschewski et al., "Circulating tumour DNA and CT monitoring in patients with untreated diffuse large B-cell lymphoma: a correlative biomarker study," Lancet Oncol. 16(5), 541-549 (2015).

41. L. Hou et al., "Blood telomere length attrition and cancer development in the normative aging study cohort," EBioMedicine 2(6), 591-596 (2015).

42. Y. Niv and A. D. Sperber, "Sensitivity, specificity, and predictive value of fecal occult blood testing (Hemoccult II) for colorectal neoplasia in symptomatic patients: a prospective study with total colonoscopy," Am. J. Gastroenterol. 90(11), 1974-1977 (1995).

43. K. Nanda et al., "Accuracy of the Papanicolaou test in screening for and follow-up of cervical cytologic abnormalities: a systematic review," Ann. Intern. Med. 132(10), 810-819 (2000).

44. R. Ferrini et al., "Screening mammography for breast cancer: American College of Preventive Medicine practice policy statement," Am. J. Prev. Med. 12(5), 340-341 (1996).

45. D. M. Eddy, "Screening for cervical cancer," Ann. Intern. Med. 113(3), 214-226 (1990).

Biographies for the authors are not available. 